\title{
Psychophysiological index attenuation to pictures
}

\author{
SPERO A. METALIS and ECKHARD H. HESS \\ University of Chicago, Chicago, Illinois 60637
}

\begin{abstract}
Young adults were shown three times in succession a series of pictures of three affective themes. Following the third presentation, a single additional novel picture, neutral in affective content, was shown. Four psychophysiological indexes were monitored: heart rate response (HR), blink rate response (BR), pupil size response (PR), and skin resistance (SR). The indexes were significantly different from the positive and negative affective themes relative to the neutral affective theme, and the subjects maintained their ability to discriminate between themes through the third presentation series, as they had through the first presentation series. Response attenuation was significant only for the SR. Baseline attenuation was greatest in the SR, intermediate in the HR and BR, and not present in the PR. The novel stimulus induced complete index dishabituation, a clear orienting response. It was concluded that psychophysiological indexes to a series of affective pictorial stimuli do not attenuate at equal rates and that attenuation is due to habituation as opposed to fatigue.
\end{abstract}

The purpose of this paper is to investigate the experimental hypothesis that different physiological response systems attenuate at different rates to pictorial stimulus repetition. Few studies have addressed the question of attenuation of psychophysiological indexes to affective pictorial stimuli (Averill, Malmstrom, Koriat, \& Lazarus, 1972; Hare, Wood, Britain, \& Shademan, 1970; Klorman, 1974; Mangelsdorff \& Zuckerman, 1975; Libby, Lacey, \& Lacy, Note 1), and their findings have not proved consistent. For example, Libby et al. presented $\mathbf{4 0}$ control slide/picture slide pairs for $15 \mathrm{sec}$ each while recording pupil and heart rate responses. The test session was divided into halves by a 5-min rest break. Analyses across subjects revealed that between the two quarters of the first half-session and the two quarters of the second half-session (i.e., during the course of each half-session), the pupil baseline decreased and the heart rate increased, whereas between the two half-sessions, the pupil baseline increased and the heart rate decreased. The authors pointed out that these findings, of sympathetic-like heart increases accompanied by parasympathetic-like pupil size decreases within half-sessions and the inverse of these changes between half-sessions, cannot be accounted for by any existing theory.

Of the above studies, none has sought to differentiate between the effects of habituation and fatigue. This confounding of causal phenomena may account for some of the disparity of findings in reports of psychophysiological index attenuation. The major aim of the present study was to distinguish between

Requests for reprints should be addressed to Spero Metalis, Department of Psychology, Loyola University of Chicago, Chicago, Illinois 60626. the effects of these two causal phenomena. In addition, the investigation yielded evidence bearing on the "bidirectionality" hypothesis (Hess, 1965) and the response patterning phenomena: situational stereotypy and directional fractionation (Lacey, 1959). Also, a relatively new statistical technique, multivariate analysis of variance (Bock, 1975), was used to deal with the simultaneously measured psychophysiological response indexes.

\section{METHOD}

\section{Subjects}

University students, 33 men and 23 women, were asked to participate in a study involving "assessment of people's reactions to emotion evoking pictures." Complete data were obtained from 26 men and 18 women. Data from the remaining subjects were limited by measurement artifact or equipment malfunction.

\section{Stimuli and Apparatus}

The stimuli consisted of a set of 15 pairs of slides. Each picture slide was preceded by a control slide of comparable luminance value. The slides were prepared in the manner described by Hess (1972) and conformed to the specifications given therein.

On the basis of their denotative content, the pictures were pooled into three thematic categories, or themes: (1) benign-four pictures of what may be regarded as normal people in neutral situations; (2) disease-four pictures of clinical photos of people with severe skin diseases; and (3) nudes-six pictures of men and/or women in what are commonly regarded as "erotic" photos. For a given subject, the nudes theme consisted of two nude couple stimuli plus two photos of nudes of the sex opposite to that of the subject. Analyses of responses to the two same-sex photos were outside the purposes of this study. An additional category was designated novel-a single, presumably neutral, picture that was made unexpected by the experimental procedure.

The stimuli were arranged to minimize the clustering of thematically similar pictures. Four different stimulus orders were given to account for possible stimulus order effects, and stimulus orders were counterbalanced. 
The slides in each control/picture pair appeared as $12 \times 18 \mathrm{~cm}$ images rear-projected onto a screen $1 \mathrm{~m}$ directly in front of the chin-and-forehead stand supporting the subject's head, so the slides subtended a visual angle of approximately $20 \mathrm{deg}$ on the horizontal plane and $15 \mathrm{deg}$ on the vertical. Each slide presentation period was $7 \mathrm{sec}$ in duration, with a 1 -sec interslide interval. Timing was accomplished via the built-in timer of the Kodak 860 Carousel projector, which was rear-projecting the slides onto the image screen. The projector lens was $120 \mathrm{~cm}$ from the back of the screen. An opaque disk with a 6-mm round hole in its center was fitted over the projector's Kodak Zoom Ektanar f3.5 lens to reduce overall illumination of the slides to between 2.4 and $6.5 \mathrm{fc}$ at the screen.

The psychophysiological indexes used and their means of transduction were: (1) Heart rate response (HR)-obtained from two aluminum mesh disk electrodes $2.5 \mathrm{~cm}$ in diameter, one affixed to each of the distal surfaces of the subject's two arms, roughly over the biceps femoris muscles. A mesh ground electrode was affixed to the forehead between and above the eyes. (2) Blink rate response (BR) and pupil size response (PR)-obtained via a Whittaker 8000 television pupillometer, which converts changes in pupil diameter as well as eye blinks into voltage changes. (3) Skin resistance response (SR)-obtained from two lead electrode plates, each $2 \times 3.5 \mathrm{~cm}$. One was affixed approximately over the second metacarpal-carpal joint and the other over the fifth metacarpal-carpal joint of the palmer surface of the nondominant hand. The electrodes were connected to a Harvard Apparatus GSR transducer set to yield "differential" galvanic skin responses. The system, therefore, recorded relative skin resistance deviations from an arbitrary baseline, the first derivative of GSR. The differential GSR was selected over the more conventional GSR index because it was a more informative and robust index, given the relatively rapid stimulus changes. Preliminary comparison tests revealed that the response time of conventional GSR was slower; this index required greater care to minimize artifactual contamination, yet it provided no compensating advantage over differential GSR in terms of information on which to base distinctions among the experimental factors.

Grass EC2 electrode cream was used on all electrodes, and they were held in place with adhesive tape.

Recording of the psychophysiological indexes was accomplished as follows: Pupillometer output was recorded on a Bausch \& Lomb VOM-5 chart recorder connected in parallel with one channel of a Grass Model 5 polygraph. The polygraph channel served as a time-event reference between the index on the polygraph chart paper and the chart recorder. The VOM-5, by permitting greater range for pen displacement on the $Y$-axis, provided greater accuracy of PR measurement than did the polygraph. The system was calibrated so that a $1-\mathrm{mm}$ difference in pupil diameter yielded a $2.5-\mathrm{cm}$ difference in VOM-5 pen deflection. Leads from the SR transducer and the HR electrodes were connected to DC preamps of the polygraph. The time constant and low-pass frequency filter settings for SR were .1 and 3, respectively, and for HR they were .8 and 15 . The amplitude of pen deflection was $5 \mathrm{~mm}$ for a $1-\mathrm{mV}$ internal (polygraph) calibration signal for the SR channel and $20 \mathrm{~mm}$ for the HR channel. Paper drive speed of the polygraph was $10 \mathrm{~mm} / \mathrm{sec}$, and, for the chart recorder, was $2.5 \mathrm{~mm} / \mathrm{sec}$.

The psychophysiological indexes were scored manually. In the HR channel, during each 7-sec slide presentation interval, the between-R-wave intervals were counted to the nearest tenth of an interval. This frequency count was converted to beats per minute before being further analyzed. Eyeblinks appeared as distinct spike-like deflections easily discriminable from PR, so BR and PR were obtained from the same channel. The number of blinks per slide was manually counted. The pupil record of each slide interval was divided into five equal periods, and the median pupil size of each was measured with a ruler to the nearest tenth of a millimeter. The average of these scores was taken as the pupil size to that slide interval. Visual examination of the SR polygraph record permitted ascertainment of a "baseline" or level of little apparent electrodermal activity/change taking place. A line was drawn through this baseline level. From this line, the extent of each of however many pen deflections present was measured in millimeters, and the sum of these scores for each slide presentation interval yielded the skin resistance change score for that slide.

Scoring of the psychophysiological index chart data was done by three scorers. Checks on reliability were made at three different points in the course of their work: soon after the beginning, toward the middle, and toward the completion of the scoring task, on each of the four indexes. Pearson correlations between the ratings of pairs of scorers of a given subject's data were always found to be greater than .90 .

A questionnaire used to determine each subject's feelings about each picture was composed of 16 items taken primarily from the semantic differential scale questionnaire used by Libby, Lacey, and Lacey (1973). A picture's interest value was determined from the items making up the factor termed "attention-interest" in that study, and its pleasantness value was determined from the items making up the factor termed "pleasantness-evaluation."

\section{Procedure}

Each subject was seated on an adjustable stool so that he could rest his head comfortably on the chin-and-forehead support. The television camera of the pupillometer was aimed at the subject's left eye. The SR, HR, and ground electrodes were then attached.

The recording system was calibrated before each subject was run; thus, sensitivity of the transducers was held constant across subjects. After clarity of the recording system had been checked, the experimenter read the instructions to the subject. These explained that the subject was to view a short series of control and picture slides and that he should refrain from making head movements, since they would degrade the quality of the pupil recording.

The 14 control/picture slide pairs were then shown in sequence (Run 1). At the end of the 14th pair, the subject was told to relax and to take his head out of the headrest. He was given a booklet with the paper-and pencil questionnaire and, after he had read the instructions, the subject was again shown each of the picture slides in sequence and was given about $2 \mathrm{~min}$ per slide to complete the questionnaire for each of the pictures. The subject was told that the purpose of the questionnaire was to assess how he felt about the pictures.

When he had completed the questionnaire, the subject was repositioned in the headrest, the recording system was checked, and the stimulus series was again presented to him while the psychophysiological indexes were recorded (Run 2). Following the last of the 14 previously presented slide pairs, an additional picture slide, neutral in thematic content and preceded by its control slide, was shown. This unexpected or novel stimulus was intended to induce dishabituation and thus make possible an empirical differentiation between the effects of habituation and of fatigue on the psychophysiological responses.

\section{RESULTS}

\section{Preliminary Analyses}

The questionnaire results indicated that the nudes theme was interesting as well as pleasant, the disease theme was interesting but unpleasant, and the benign theme was uninteresting and intermediate in pleasantness. The details of this analysis are outside the purposes of this report (see Metalis, 1978).

The shapes of the psychophysiological data distributions across subjects were examined to insure that they were approximately Gaussian in shape, so 
that the assumptions of the inferential parametric statistics that were subsequently used to analyze the data would not be violated. The distribution of HR, which was skewed, was normalized through a log transformation of the data. The distribution of PR, which was leptokurtic, was normalized through an arcsine transformation.

The psychophysiological response lability and the tonic baseline level of the different subjects varied greatly. To minimize the possibility of idiosyncratic effects (Lacey, 1956) contaminating the analyses, the untransformed data of each subject were placed on an arbitrary or "pure thematic effects" scale, whose zero point was set at each subject's average psychophysiological index response across all stimuli per run and whose units of measure were the standard deviations of the individual index responses from this mean. Thus, the problems of differences between subjects in psychophysiological response lability and tonic baseline levels were dealt with by transforming each subject's data scores into z-scores. Furthermore, because the responses of four stimuli were averaged into each theme, theme response variability was reduced and the effects on psychophysiological index responses of idiosyncratic stimulus features, such as brightness contrast effects on PR, were effectively neutralized.

It should be noted here that the basis of most of the following comparisons is not the difference score between an index's value to a picture slide minus its value to a control slide. Such differences (change scores) are indeed the response values to the stimuli. But the critical comparisons are between the averaged responses to the different picture themes, the neutral benign theme in comparison with the two more affective themes.

\section{Substantive Questions}

The results of the data analyses and the subsequent discussion are centered around three questions, and these will be dealt with in turn. In the first section, it is determined that the psychophysiological indexes do indeed respond to the affective content of the picture themes. In the second section, the nature of attenuation of the indexes is examined. In the third section, the extent to which the attenuation is due to habituation, as opposed to fatigue, is assessed.

How well do the psychophysical indexes serve to discriminate between the experimental factors? The magnitudes of response differences between themes comprise the values contrasted by this first data analysis. The data were analyzed via the MESA99 program (Finn, 1972) by multivariate analysis of variance (MANOVA) in a theme $\times$ sex $\times$ run factorial design, with repeated measures on theme and run. MANOVA is the multivariate analog of univariate analysis of variance (Bock, 1975; Finn, 1974; Harris, 1975). The MANOVA takes into ac- count the interrelations between a set of related response variables by such as the simultaneously measured indexes by calculating least squares estimates of the variable set matrix. Then, multivariate $F$ ratios of the between-treatments variance over the withintreatments variance are computed, and these, along with their related probability values, are used to determine whether or not the null hypothesis should be rejected. This is preferable to conducting a separate analysis on each of several simultaneously measured variables, since this latter procedure requires the questionable assumption that the variables are independent. Also, since each of several univariate tests (one for each dependent variable) is designed to produce a significant result $a \times 100 \%$ of the time (where $a$ is the significance level of the test) when the null hypothesis is correct, the probability of having at least one of the tests produce a significant result when in fact nothing but chance variation is going on increases rapidly as the number of tests increases. The MANOVA technique provides a means of controlling this experimentwise error rate.

The least square estimates of effects, their standard error values, and the related F-test results are shown in Table 1. Order of inclusion of the indexes into the computation was HR, BR, PR, and SR, and contrasts were made orthogonal to each other.

As can be seen in Table 1, the indexes discriminate among picture themes on the basis of the their denotative meanings, that is, between the benign (B) vs. disease (D) and nudes $(N)$ themes $(B * D$ and $B * N)$. The most clearly significant difference was that of the theme main effects $(p<.0001)$.

The univariate Fs making up this overall effect indicate that the psychophysiological indexes differ in the extents of their contributions to the overall between-theme discrimination. The index that proved most discriminative was the PR, which had the largest effects in both the $B * D$ and the $B * N$ contrasts. Furthermore, the $B * D$ contrast was negative (the pupil constricted to the disease theme), whereas the $\mathrm{B} * \mathrm{~N}$ contrast was positive (the pupil dilated to the nudes theme). The PR was the only index that responded bidirectionally to the two affective themes. The HR showed a significant decrease to both; the $B{ }^{*} D$ and the $B * N$ contrast effects were negative. The BR and SR showed significant effects only to the $B * N$ contrast. The BR decreased and the SR increased to the nudes theme.

The significant multivariate $F$ for the sex effect in Table 1 is due mainly to the contributions of HR and PR differences between the sexes. The betweensexes contrast effects for $H R$ and $P R$ are significant, but they are-small; $M * W: H R$ is .77 , and $M * W$ : $P R$ is -.52 , so men had slightly more negative $H R$ responses and more positive $P R$ responses than did women. Since the index baselines of men and women were equated through the $\mathrm{z}$-score transfor- 
Table 1

MANOVA Results

\begin{tabular}{|c|c|c|c|c|c|c|}
\hline \multirow{2}{*}{\multicolumn{3}{|c|}{$\begin{array}{l}\text { MANOVA Test of Significance of } \\
\text { Psychophysiological Index Responses }\end{array}$}} & \multicolumn{4}{|c|}{ Contribution of Each Index to the Overall Value } \\
\hline & & & & Least Square & & \\
\hline Effect & $\begin{array}{l}\text { Multivariate } \\
\mathrm{F}\end{array}$ & df & PSI Contrast & $\begin{array}{l}\text { Estimate } \\
\text { of Effect }\end{array}$ & $\begin{array}{c}\mathrm{SE} \text { of } \\
\text { Estimate }\end{array}$ & $\begin{array}{c}\text { Univariate } \\
\mathrm{F}\end{array}$ \\
\hline Theme & $12.85 \ddagger$ & 8,35 & $\begin{array}{l}B * D / H R \\
B * N / H R \\
B * D / B R \\
B * N / B R \\
B * D / P R \\
B * N / P R \\
B * D / S R \\
B * N / S R\end{array}$ & $\begin{array}{r}-2.12 \\
-2.61 \\
.80 \\
-5.63 \\
-4.25 \\
5.81 \\
1.00 \\
2.44\end{array}$ & $\begin{array}{r}.99 \\
.84 \\
1.39 \\
1.04 \\
.92 \\
1.01 \\
1.02 \\
.98\end{array}$ & $\begin{array}{c}4.61 \dagger \\
9.64 \dagger \dagger \\
.33 \\
29.49 \neq \\
21.31 \neq \\
33.14 \neq \\
.96 \\
6.18 \dagger\end{array}$ \\
\hline Sex & $4.11 \dagger \dagger$ & 4,39 & $\begin{array}{l}\mathrm{M} * \mathrm{~W} / \mathrm{HR} \\
\mathrm{M} * \mathrm{~W} / \mathrm{BR} \\
\mathrm{M} * \mathrm{~W} / \mathrm{PR} \\
\mathrm{M} * \mathrm{~W} / \mathrm{SR}\end{array}$ & $\begin{array}{r}.77 \\
-.01 \\
-. .52 \\
-1.15\end{array}$ & $\begin{array}{l}.32 \\
.37 \\
.27 \\
.32\end{array}$ & $\begin{array}{l}5.18 \dagger \\
3.36 \\
4.34 \dagger \\
1.15\end{array}$ \\
\hline Run & 1.99 & 4,39 & Run $1 *$ Run $2 \neq \neq / S R$ & .86 & .42 & $4.11 \dagger$ \\
\hline Run by Theme & 2.00 & 8,35 & $\begin{array}{l}\text { Theme } * \text { Run } \neq \neq / \text { SR } \\
\text { Theme * Run/SR }\end{array}$ & $\begin{array}{l}1.73 \\
2.17\end{array}$ & $\begin{array}{l}.89 \\
.91\end{array}$ & $\begin{array}{l}3.79 \dagger \\
5.72 \dagger\end{array}$ \\
\hline Sex by Theme & 1.00 & 8,35 & & & & \\
\hline Sex by Run & 1.18 & 4,39 & & & & \\
\hline Sex by Run by Theme & .74 & 8,35 & & & & \\
\hline
\end{tabular}

Note $-B=$ benign theme, $D=$ disease theme, $N=$ nudes theme; $M=$ men, $W=$ women; $H R=$ heart rate response, $B R=$ blinkrate response, $S R=$ skin response. MANOVA $=$ theme $\times \operatorname{sex} \times$ run $\left(3^{*} 2^{*} 2\right)$ with repeated measures on run and theme.

$\dagger p<.05 . \quad \dagger \neq p<.01 . \quad \neq p<.0001 . \quad \neq \neq$ Univariate contribution noted despite nonsignificant multivariate effect.

mation, these between-sexes response differences reflect sex differences in the direction of response.

Although the evidence indicates that there is some difference between the sexes in their overall response pattern, the MANOVA results displayed in Table 1 show that there are essentially no sex-related differences in attenuation of response patterns of subjects to the picture themes. That is, the interaction effects are nonsignificant.

Patterning of autonomic indexes, situational stereotypy (Lacey, 1959), has been reported to occur as a result of task demands of the stimulus situation on the subject. Because the various picture themes elicited distinctive psychophysiological patterns, the data suggest the presence of the situational stereotypy phenomenon. In order to determine how widespread the patterning phenomenon was, a nonparametric statistical analysis of the data was conducted. For each subject, each psychophysiological index was ranked in order of magnitude across the three themes. That theme for which an index was largest was ranked as 1 , that theme for which the index was smallest was ranked as 3, and that theme for which the index was intermediate was ranked as 2 . For each index, the number of instances of all three rankings proving identical between the two runs, of only one ranking in common between the runs, and of no rankings in common between the runs was obtained from the 44 subjects who had complete data. On the basis of chance, $1 / 6 \times 44$, or 7 , subjects should have displayed identical between-runs rankings, $1 / 2 \times 44$, or 22 , should have displayed one ranking in common, and $1 / 3 \times 44$, or 15 , should have displayed no rankings in common. In the case of the HR, the obtained frequencies were 12,18 , and 14 , respectively. Likewise, for the BR they were 16,17 , and 11 , for the PR they were 13, 20, and 6, and for the SR they were 5,22 , and 17 . Chi-square tests were done comparing the observed with the expected frequencies. Given two degrees of freedom, findings significantly different from chance were indicated for the BR $\left(\chi^{2}=12.64, \mathrm{p}<.005\right)$ and the PR $\left(\chi^{2}=10.72\right.$, $\mathrm{p}<.005)$, but not for the HR $\left(\chi^{2}=4.37, \mathrm{p}<.250\right)$ or the SR $\left(\chi^{2}=.84, \mathrm{p} \cong .50\right)$. Therefore, response patterning to the themes between runs for a given subject (i.e., situational stereotypy of the run effects) was clearly present in the case of the BR and PR but nonsignificant in the case of the HR and the SR.

For each psychophysiological index, the frequency of occurrence of 1 rankings under each of the three themes, for each run separately, was computed in order to examine situational stereotypy among the indexes to the theme effects. Table 2 shows the tabulations across subjects.

On the assumption of equal probabilities of occurrence of 1 rankings, chi-square tests of significance were computed for each theme. Clearly, 1 rankings are not uniformly distributed. For the PR index, there is a greater number of 1 rankings under the nudes theme in both runs, confirming that the larg- 
Table 2

Frequency Counts of First Rankings and Third Rankings of Each Psychophysical Index for Each Theme Across Subjects

\begin{tabular}{|c|c|c|c|c|c|c|c|c|}
\hline \multirow[b]{2}{*}{ PSI } & \multicolumn{4}{|c|}{ Run 1} & \multicolumn{4}{|c|}{ Run 2} \\
\hline & B & $\mathrm{D}$ & $\mathrm{N}$ & $x^{2}$ & B & $\mathrm{D}$ & $\mathrm{N}$ & $x^{2}$ \\
\hline & \multicolumn{8}{|c|}{ First (1) Rankings } \\
\hline PR & 8 & 7 & 29 & $20.6 \dagger$ & 7 & 4 & 33 & $33.9+$ \\
\hline SR & 7 & 18 & 19 & $5.9 *$ & 13 & 13 & 18 & 1.1 \\
\hline HR & 25 & 9 & 10 & $10.7 * *$ & 23 & 10 & 11 & $6.8 *$ \\
\hline \multirow[t]{2}{*}{ BR } & 13 & 14 & 7 & 4.6 & 20 & 19 & 7 & $7.0^{*}$ \\
\hline & \multicolumn{8}{|c|}{ Third (3) Rankings } \\
\hline PR & 22 & 18 & 4 & $11.9 * *$ & 18 & 24 & 2 & $16.7 \dagger$ \\
\hline SR & 22 & 11 & 11 & 5.4 & 13 & 17 & 14 & .67 \\
\hline HR & 9 & 20 & 15 & 4.1 & 12 & 18 & 14 & 1.33 \\
\hline $\mathrm{BR}$ & 8 & 11 & 25 & $11.0 * *$ & 8 & 13 & 23 & $7.8 *$ \\
\hline
\end{tabular}

Note-A chi-square one-sample test was used. $B=$ benign, $D=$ disease, $N=$ nudes. $\quad{ }^{*} p<.05 . \quad{ }^{* *} p<.01 . \quad t_{p}<.001$.

est PRs most often occurred to the nudes theme. The table shows that the largest SRs most often occurred to the disease and nudes themes, but the effect is not significant by Run 2. The significant chisquare score for the HR is due to the high frequency of 1 rankings under the benign theme. However, the largest HR decrement responses are reflected by the smallest rank order scores, 3 rankings. An examination of the frequency of occurrence of 3 rankings, greatest HR decreases, shows that they occurred most frequently to the disease theme, second most to the nudes theme, and least to the benign theme. The BR is likewise an index in which response decreases were informative; the chi-square analysis showed a significantly larger number of 3 rankings to the nudes theme.

In summary, then, the nonparametric analysis results generally confirmed those of the parametric analysis concerning central tendencies of run and theme effects, and further showed that theme-induced response patterning was quite widespread among the subject sample.

Is there attenuation of the psychophysiological indexes in ability to discriminate among themes or in baseline level from Run 1 to Run 2? To what extent is each individual index affected? The results of the MANOVA, shown in Table 1, indicate that there was no significant multivariate difference between
Run 1 and Run 2. This holds for the main run effect and its higher order interactions. However, in the run effect, the univariate Fs indicated that only the SR contrast was significant (i.e., SR changed significantly between runs). The multivariate run $x$ theme effect approximated significance because of the significant contribution of the SR for both SR contrasts, as noted in Table 1.

The basis for this effect is shown in Table 3, which displays the untransformed mean psychophysiological values to each of the themes of each of the runs across sexes. The baseline attenuation of the SR index between Run 1 and Run 2 is striking. But the critical difference is that whereas in Run 1 the SR to the disease and nudes pictures was greater than that to the control slides, the opposite was the case in Run 2. The changes between Run 1 and Run 2 response values of the other indexes were not as extreme. This reflects the fact that their ability to discriminate among the denotative meanings of the three themes had not been reduced significantly. To all themes in both runs, the HR and BR responses were ones of rate decrease. The $P R$ showed some diameter decrease to the benign theme, greater decrease to the disease theme, and diameter increase to the nudes theme.

Table 3 indicates that, with the exception of the SR, there were no marked baseline changes of averaged theme scores from Run 1 to Run 2. But, since subjects had been counterbalanced with respect to stimulus order to prevent confounding of theme with order of stimulus sequence effects, theme averages obscure possible within-runs and between-runs changes in baselines. Therefore, the untransformed control slide psychophysiological index baseline values to the 1st, 5th, 10th, and 14th control/picture slide pairs, the benign theme, were averaged across subjects. The results are shown in Figure 1. Because each subject was shown one of four different possible slide orders, any one of the four benign control/ picture slide pairs could serve as the 1st, 5th, 10th, and 14th stimulus. Thus, a response component due to an idiosyncratic feature of any one control slide, such as its luminance value, was equally distributed among the four stimulus positions shown in Figure 1.

Within runs, one apparent pattern is that of sympathetic-like arousal on the first and last of the slides,

Table 3

Untransformed Psychophysiological Index Averaged per Run per Theme per Slide

\begin{tabular}{|c|c|c|c|c|c|c|c|c|c|c|c|c|}
\hline \multirow[b]{3}{*}{ Index } & \multicolumn{6}{|c|}{ Run 1} & \multicolumn{6}{|c|}{ Run 2} \\
\hline & \multicolumn{2}{|c|}{ Benign } & \multicolumn{2}{|c|}{ Disease } & \multicolumn{2}{|c|}{ Nudes } & \multicolumn{2}{|c|}{ Benign } & \multicolumn{2}{|c|}{ Disease } & \multicolumn{2}{|c|}{ Nudes } \\
\hline & $\mathrm{C}$ & $\mathbf{P}$ & $\mathrm{C}$ & $\mathbf{P}$ & $\mathrm{C}$ & $\mathbf{P}$ & $\mathrm{C}$ & $\mathbf{P}$ & C & $\mathbf{P}$ & $\mathrm{C}$ & $\mathbf{P}$ \\
\hline HR & 79.60 & 78.90 & 78.90 & 77.00 & 80.20 & 78.40 & 78.70 & 78.70 & 78.20 & 77.90 & 79.30 & 78.50 \\
\hline BR & 2.10 & 1.20 & 2.40 & 1.30 & 2.30 & .70 & .2 .30 & 1.50 & 2.30 & 1.20 & 2.50 & 1.00 \\
\hline PR & 5.01 & 4.95 & 4.99 & 4.91 & 5.02 & 5.14 & 5.03 & 5.01 & 5.00 & 4.91 & 5.02 & 5.13 \\
\hline SR & 1.79 & 1.41 & 1.45 & 2.09 & 1.55 & 1.92 & .84 & .77 & .93 & .61 & .77 & .49 \\
\hline
\end{tabular}

Note $-C=$ control slide, $P=$ picture slide. 


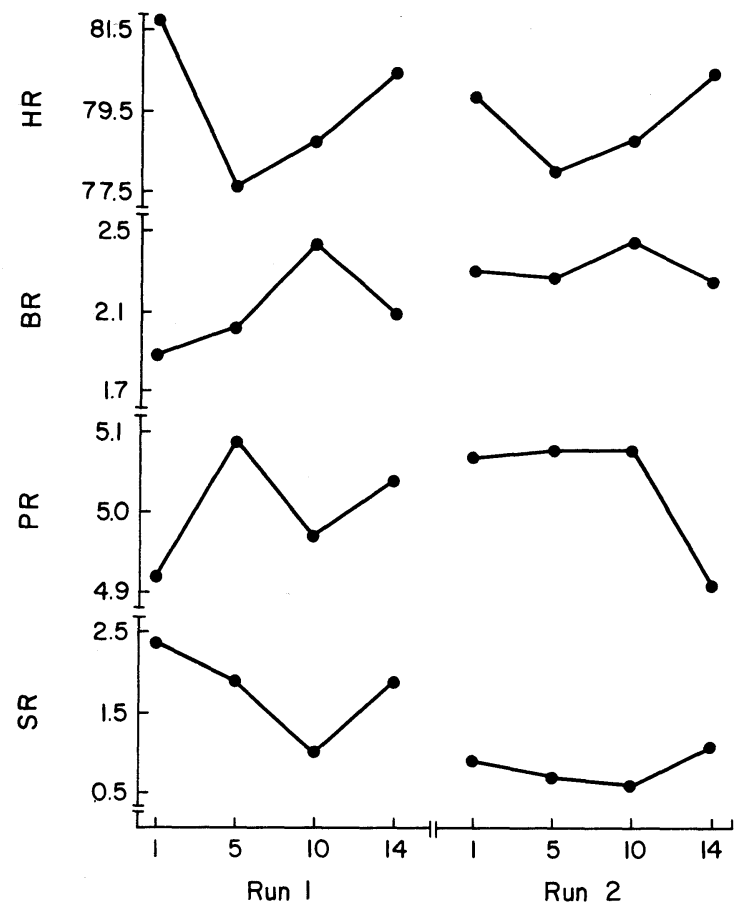

Figure 1. Psychophysiological index baseline changes as depicted by control slide values, averaged across subjects, to the 1st, 5th, 10th, and 14th stimuli in each of the two runs. The heart-rate response (HR) is in beats per minute, the blink rate response (BR) is in blinks per slide interval $(7 \mathrm{sec})$, the pupil size response (PR) is the average diameter in millimeters per slide interval, and skin response (SR) is in centimeters of polygraph pen deflection per slide interval.

relative to the baseline levels of the middle slides. This is most apparent in the case of the HR and SR, less so in the case of the BR, and least in the case of the PR.

Between runs, the most noticeable pattern that can be seen in Figure 1 is that, of all the indexes, the SR displayed the greatest baseline attenuation between Run 1 and Run 2. All four data scores on Run 2 fall below the Run 1 values. In the case of the BR, all four data scores on Run 2 are above the Run 1 values, while only three of the four HR scores show this relationship. In these two indexes, attenuation was manifested by an increase in baseline rate. There was no noticeable difference between Run 1 and Run 2 in the case of the PR.

In order to test the significance of these apparent baseline changes, two-factor (run by stimulus sequence) within-subjects analyses of variance were run on the untransformed benign theme control slide baseline values of each psychophysiological response index. In order to determine the shape of the curves that best fit the data points of the various indexes, the factors were subdivided into trend components by use of orthogonal polynomials. The MESA99 program treats the main effects and their interactions as multivariate variables and the trend components (linear, quadratic, and cubic) as univariate components of these variables.

The sequence factor alone was significant in the case of the HR baseline $[F(3,39)=6.13, p<.02]$, and the trend analysis revealed that these data are best characterized by a quadratic function $[F(1,39)$ $=16.48, \mathrm{p}<.001]$. As is shown in Figure 1, the second and third values in the sequence are lower than the first and last values. The run $\times$ sequence interaction effect proved nonsignificant; the sequence pattern is the same in both runs.

In the case of the BR baseline, the results indicate that, although the difference between runs was not significant, the within-runs sequence effect was significantly different $[F(3,39)=3.94, p<.02]$. The orthogonal polynomial analysis indicated that a quadratic function best describes the distribution of values $[F(1,39)=8.87, p<.005]$. This supports the appearance of an inverted U-shaped curve described by the data points in Figure 1. The run $\times$ sequence interaction effect was nonsignificant.

For the PR baseline, there was no significant attenuation between or within runs; the run and the sequence effects were nonsignificant. However, the run $\times$ sequence interaction effect was significant, because the sequence pattern in Run 1 was different from that in Run 2. A cubic function best characacterizes the sequence pattern in Run $1[F(1,35)=$ $12.15, \mathrm{p}<.001]$. while a linear function best characterizes the pattern in Run $2[F(1,35)=10.06, p<$ .003 ].

Of the four psychophysiological indexes, only the SR yielded a significant run effect $[F(1,41)=14.85$, $\mathrm{p}<.005]$. This is the only index whose baseline was significantly lower in Run 2 than in Run 1. Within each run, the SR values did not remain unchanged; the sequence factor was significant $[F(3,39)=3.94$, $\mathrm{p}<.02$ ]. The test of trend revealed that this withinruns change is best characterized by a quadratic function $[F(1,39)=8.87, p<.005]$, as was the case with the HR and BR. This confirms what can be seen in Figure 1. The second and third values are lower than the first and last values in the sequence.

To what extent is the psychophysiological index attenuation due to habituation and/or fatigue? As was explained in the introduction, this question was experimentally dealt with via the novelty paradigm. At the end of Run 2, a novel stimulus of neutral thematic content that was expected to elicit dishabituation of the psychophysiological indexes was presented.

For purposes of comparison, the untransformed index responses across all subjects to the novel control/picture slide pair, the 10th control/picture slide pair shown on Run 1 , and the 5th control/picture slide pair shown on Run 2 were analyzed. These stimuli were all neutral in thematic content-they were from the benign theme. These were selected 
Table 4

ANOVA of Psychophysiological Index Responses to Discriminate Between Attenuation Effects of Habituation vs. Fatigue

\begin{tabular}{|c|c|c|c|c|c|}
\hline \multirow[b]{3}{*}{ Index } & \multicolumn{3}{|c|}{$\begin{array}{l}\text { Mean Response to Each } \\
\text { of Three Stimuli }\end{array}$} & \multirow[b]{3}{*}{$\mathrm{df}$} & \multirow[b]{3}{*}{$\mathrm{F}$} \\
\hline & \multicolumn{2}{|c|}{ Neutral } & \multirow[b]{2}{*}{ Novel } & & \\
\hline & Run 1 & Run 2 & & & \\
\hline HR & -.38 & -.79 & -1.39 & 2,86 & .65 \\
\hline BR & -1.35 & -.93 & -.50 & 2,104 & 2.70 \\
\hline PR & -.07 & -.11 & .08 & 2,98 & $11.45^{*}$ \\
\hline SR & .28 & -.20 & 1.21 & 2,102 & $7.30^{*}$ \\
\hline
\end{tabular}

${ }^{*} p<.001$.

because it was necessary to choose different neutral stimuli from the two runs to permit between-stimuli statistical comparisons with the novel stimulus.

A univariate analysis of variance was done, with each psychophysiological index separately serving as the dependent variable, and the neutral stimulus of Run 1, the neutral stimulus of Run 2, and the novel stimulus serving as the three within-subjects treatments. In addition, the possible effect of sex, a between-subjects factor, was tested. However, because the sex factor proved nonsignificant, it was not displayed in the table of results. The results, shown in Table 4, indicate significant PR and SR effects due to the increase in these indexes to the novel stimulus. The HR to this stimulus decreased, relative to the other two, but this effect was not significant. The BR decrease was greatest to the first stimulus, intermediate to the second, and least to the novel stimulus, a pattern unlike that of the other indexes. In addition, for each index, a pairwise $t$ test was run between the novel picture slide value and its control slide value. Given the degrees of freedom, 54, all indexes yielded responses significant at or beyond the .05 level. Specifically, to the novel picture slide, the HR displayed rate decrease $(t=-2.15)$, as did the $B R(t=-2.33)$; the PR displayed dilation $(t=3.48)$. The strongest activation was yielded by the $S R$ index $(t=3.48)$.

\section{DISCUSSION}

The experiment provided answers to three sets of questions. First, it was found that the psychophysiological indexes discriminated clearly among the three picture themes. The PR proved to be the most discriminative index, and it was the only one that responded bidirectionally. The response differences between the sexes were minor, and they proved unrelated to the theme or run effects. Relative"to the benign theme, the nudes theme, which was rated as very interesting as well as pleasant, elicited pupillary dilation, an increase in SR, and rate decreases in BR and HR. The disease theme, which was rated as interesting but unpleasant, elicited pupillary constriction.
The other indexes responded in the same direction to the disease theme as they did to the nudes theme, but magnitudes of their responses proved nonsignificant to the disease theme.

The findings concerning the PR support the Hess (1965) bidirectionality hypothesis. The pupil responded with dilation to the pleasant stimulus theme and with constriction to the unpleasant stimulus theme, despite the fact that they were both interesting. The results of the analysis of smallest rank order values indicate that the $P R$ is the only index that apparently fails to confirm the conclusions arrived at by the MANOVA parametric statistical analysis. Table 2 shows that although the chi-square value was significant in the case of 3 rankings in PR, the greatest frequency of small PR values (including constrictions) was to the benign and disease theme. In Run 1, there was a greater number of 3 rankings to the benign theme, whereas by Run 2, the greatest number of 3 rankings was to the disease theme. These results, in conjunction with the MANOVA finding of a significant negative $B * D$ contrast effect in both runs, indicate that, in Run 1, the constriction PR to the disease theme, although significant, is not as widespread as it is by Run 2. Constrictions to the disease theme are more frequent in Run 2, perhaps due to attenuation of the initial shock value of this theme. The shock would tend to elicit a PR dilation effect. This interpretation of these PR findings is congruent with the bidirectionality hypothesis (Hess, 1965) and its subsequent elaboration on the topic of shock-evoking stimuli (Hess, 1975, p. 49).

The HR varied with the affective themes' interest values; relative to the benign theme, the other two themes were rated as more interesting, and they elicited greater HR decreases. This finding tends to support the hypothesis that attention to external stimuli is directly related to HR diminution (Lacey, 1959). The BR and SR indexes were affected most by the nudes theme, the theme that was high in both interest and pleasantness value, so they were indexing nonspecific cognitive-affective content. The finding that blinking occurs more frequently during control slides than during picture slides corroborates the findings of Marais (1974). Holland and Tarlow (1975) suggested that blinking is timed so as not to interfere with significant visual input. This hypothesis is supported by the present findings. Not only did BR decrease to picture, relative to control, slides, but also the decrease to the nudes theme was significantly greater than that to the benign theme. Thus, BR decrease was greater to the more significant visual stimuli.

Each stimulus theme elicited a distinctive psychophysiological pattern. Furthermore, sympathetic-like activity in one index occurred simultaneously with parasympathetic-like activity in another index (e.g., PR dilation with HR diminution). Of these findings, 
the former is an instance of the phenomenon of situational stereotypy, and the latter is an instance of the phenomenon of directional fractionation (Lacey, 1956, 1959). That these well-documented patterning phenomena were evidenced lends support to the validity of this study's experimental methods.

Second, it was found that the psychophysiological indexes as a group did not have their themediscriminating abilities diminished significantly by the third presentation of the stimulus sequence, on Run 2. The only index that was significantly attenuated by Run 2 in its theme-discriminative abilities was the SR. The baselines of the psychophysiological indexes also did not attenuate in unison. The SR again was the index that attenuated the most between runs, the BR and $H R$ attenuated to a nonsignificant degree, and the PR did not attenuate at all.

Sokolov $(1960,1963)$ studied the orienting response, utilizing several response measures, including galvanic skin response, heart rate, and the pupil response. He found that if a tone or light stimulus is repeatedly presented, there is an initial activation of the orienting response index, followed by a return to baseline with repeated stimulus presentation. He did not test all the response indexes simultaneously, but each individually responded with this same pattern.

The present data indicate that the presentation of a series of picture stimuli also is accompanied by initial activation followed by attenuation of the orienting response indexes. However, all the components of the orienting response do not attenuate in unison. Some indexes attenuate more rapidly than others to pictorial stimuli. Because the series of picture stimuli is complex, unlike those used by Sokolov, each psychophysiological index may selectively habituate to that set of stimulus features to which it is most responsive.

The lack of a decrease in pupillary responsivity is in accord with the findings of Clynes (1962), but this finding, as well as the lack of a decrease in pupil baseline level, is discrepant with others' findings. Most investigators of pupillary responses to pictorial stimuli have reported baseline attenuation (Hess, 1972; Lehr \& Bergum, 1966; Peavler, 1974; Woodmansee, Note 2). This discrepancy may be due to the types of stimuli used. Hess (1975) reported no attenuation in pupil responsivity of subjects viewing television films, even after half an hour. Of the studies reporting attenuation of baseline pupil size, only Libby, Lacey, and Lacey (Note 1) used a wide variety of affective picture stimuli, and they did not find a simple decrease in pupil size over the time of the experimental session. Other types of stimuli more limited in variety may be less interesting to the subjects. In the terminology of Sokolov (1963), the presentation of a series of similar pictures would enhance the probability of agreement between incoming stimulus parameters and the existing neural model and thus facilitate habituation of the PR component of the orienting response. A series of dissimilar pictures would tend to present stimulus parameters that did not correspond to the neural model, and hence habituation would be retarded. The specific stimulus parameters that maintain the responsivity of the pupil more than of the other indexes remain to be determined, however.

Apparently, subjects were more "vigilant" toward the beginning and the end of each run than they were in the middle, as indicated by the fact that all the indexes except the PR showed some form of U-shaped baseline curve within sessions. This is probably due to the instructions given to the subjects prior to experiment onset. They were told that they would be shown 14 pictures, and the within-runs pattern of the indexes suggests that the subjects kept count of the stimuli. The heightened baselines at the onset of the session may have reflected anticipation concerning the nature of the stimuli that were to follow. By the middle of the session, the baseline levels waned because the nature of the stimuli was now known. At the end of the session, the baseline levels increased again in anticipation of the unknown events that would follow. Possibly, the effects of the vigilance phenomenon on the PR were small, and they were swamped by other features of the stimuli to which the PR was more sensitive.

Third, it was found that, relative to the neutral stimuli in Run 1 and Run 2, the psychophysiological index response pattern to the novel stimulus was characterized by HR diminution, PR dilation, and $S R$ activation, and a less than usual BR rate decrease. This latter score is probably due to a tendency to blink in response to the novel picture just after its onset, as if it elicited a mild startle response.

These findings point to an interesting conclusion. In comparison with the responses to the other two stimuli, the psychophysiological index responses to the novel stimulus were generally indicative of the orienting response that characterized the dishabituation phenomenon described by Sokolov (1963). Thus, any response attenuation in Run 2 must be due solely to habituation, for if fatigue were the cause, psychophysiological indexes could not respond to the novel stimulus as strongly as they responded to the Run 1 stimulus. As it was, the response to the novel stimulus was stronger than the response to the Run 1 benign stimulus, indicating that the unexpectedness of the novel stimulus elicited responsiveness over and above that of viewing a neutral stimulus for the first time.

It seems likely, then, that those studies that have reported "fatiguing" of the pupil response with repeated stimulations (e.g., Lowenstein \& Loewenfeld, $1952 \mathrm{a}, 1952 \mathrm{~b})$ were actually evidencing response attenuation due to habituation and were mislabeling the phenomenon. However, a tonic pupil size de- 
crease may be a corollary of an overall state of physical fatigue (Lowenstein, Feinberg, \& Loewenfeld, 1963). This possibility has yet to be adequately documented as a phenomenon distinct from that induced by habituation.

\section{REFERENCE NOTES}

1. Libby, W. I., Jr., Lacey, B., \& Lacey, J. I. Phasic and tonic pupillary responses to pictorial stimuli. Paper presented at the 80th Annual Meeting of the American Psychological Association, Honolulu, Hawaii, September 1972.

2. Woodmansee, J. J. Pupil reaction as an index of positive and negative affect. Paper presented at the 75th Annual Meeting of the American Psychological Association, Washington, D.C., 1967.

\section{REFERENCES}

Averill, J. R., Malmstrom, E. J., Doriat, A., \& Lazarus, R. S. Habituation to complex emotional stimuli. Journal of Abnormal Psychology, 1972, 80, 20-28.

Bock, R. D. Multivariate statistical methods in behavioral research. New York: McGraw-Hill, 1975.

Clynes, M. The non-linear biological dynamics of unidirectional rate sensitivity illustrated by analog computer analysis. Pupillary reflex to light and sound, and heart rate behavior. Annals of the New York Academy of Sciences, 1962, 98, 806-844.

FinN, J. D. Multivariance: Univariate and multivariate analysis of variance, covariance and regression. Chicago: International Educational Services, 1972.

Hare, R. D., Wood, K., Britain, S., \& Shadman, J. Autonomic responses to affective visual stimulation. Psychophysiology, $1970,7,408-417$.

HARRIS, R. J. A primer of multivariate statistics. New York: Academic Press, 1975.

Hess, E. H. Attitude and pupil size. Scientific American, 1965, 212, 46-54.

Hess, E. H. Pupillometrics. In N. Greenfield \& R. Sternbach (Eds.), Handbook of psychophysiology. New York: Holt, Rinehart \& Winston, 1972.

Hess, E. H. The tell-tale eye. New York: Van Nostrand Reinhold, 1975.

Holland, M. K., \& Tarlow, G. Blinking and thinking. Perceptual and Motor Skills, 1975, 41, 403-406.
KLORman, R. Habituation of fear: Effects of intensity and stimulus order. Psychophysiology, 1974, 11, 15-26.

LACEY, J. I. The evaluation autonomic responses: Toward a general solution. Annals of the New York Academy of Sciences, 1956, 67, 123-164.

LACEY, J. I. Psychophysiological approaches to the evaluation of psychotherapeutic process and outcome. In E. A. Rubinstein \& M. B. Parloff (Eds.), Research in psychotherapy. Washington, D.C: American Psychological Association, 1959.

LEHR, D. J., \& Bergum, B. O. Note on pupillary adaptation. Perceptual and Motor Skill, 1966, 23, 917-918.

Libby, W. I., JR., Lacey, B. C., \& Lacey, J. I. Pupillary and cardiac activity during visual attention. Psychophysiology, 1973, 10, 270-294.

Lowenste in, O., Feinberg, R., \& Lowenfeld, I. E. Pupillary movement during acute and chronic fatigue. Investigative Ophthalmology, 1963, 2, 138-157.

LoWenstein, O., \& Lowenfeld, I. E. Disintegration of central autonomic regulation during fatigue and its reintegration by psychosensory controlling mechanisms: I. Disintegration. Pupillographic studies. Journal of Nervous and Mental Disease, $1952,115,1-21$. (a)

Lowenstein, O., \& Lowenfeld, I. E. Disintegration of central autonomic regulation during fatigue and its reintegration by psychosensory controlling mechanisms: II. Reintegration. Pupillographic studies. Journal of Nervous and Mental Disease, 1952, 115, 121-145. (b)

Mangelsdorff, A. D., \& Zuckerman, M. Habituation to scenes of violence. Psychophysiology, 1975, 12, 124-129.

Marais, H. C. The eye blink as artifact in pupillometric research. Perceptual and Motor Skills, 1974, 39, 224-226.

Metalis, S. A. Psychophysiological responsivity to affective pictures (Doctoral dissertation, University of Chicago, 1978). Dissertation Abstracts International, 1978, 39, 430B.

Peavler, W. S. Individual differences in pupil size and performance. In M. P. Janisse (Ed.), Pupillary dynamics and behavior. New York: Plenum Press, 1974.

Soxolov, E. N. Neuronal models and the orienting reflex. In M. A. B. Brazier (Ed.), The central nervous system and behavior. New York: Josiah Macy, Jr., Foundation, 1960.

Sokolov, E. N. Perception and the conditioned reflex. New York: Pergamon Press, 1963.

(Received for publication May 21, 1979; revision accepted May 15, 1981.) 